\title{
Pancreatic metastasis resulting from thymic neuroendocrine carcinoma: A case report
}

\author{
YANG DU $^{1 *}$, YING WANG $^{2 *}$, JIE TANG $^{1}$, JUN GE $^{1}$, QING QIN $^{1}$, LI JIANG $^{1}$, \\ XIAOKE LIU ${ }^{1}$, XIANGLAN ZHU ${ }^{3}$ and YONGSHENG WANG ${ }^{1}$

\begin{abstract}
${ }^{1}$ Department of Medical Oncology, Cancer Center, State Key Laboratory of Biotherapy, West China Hospital, Sichuan University, Chengdu, Sichuan 610041; ${ }^{2}$ Department of East Ward Oncology, Sichuan Academy of Medical Sciences and Sichuan Provincial People's Hospital, Chengdu, Sichuan 610072; ${ }^{3}$ Department of Pathology, West China Hospital, Sichuan University, Chengdu, Sichuan 610041, P.R. China
\end{abstract}

Received February 26, 2015; Accepted December 18, 2015

DOI: $10.3892 / 01.2016 .4112$

\begin{abstract}
Thymic neuroendocrine carcinoma (NEC) is a rare type of cancer. Unlike other thymic epithelial tumors and carcinoids originating in other locations, thymic NEC possesses a more aggressive biological behavior, including invasion to proximal structures, local recurrence and distant hematogenous metastasis. Distant metastasis is often observed in the bones, lungs, spleen, liver and adrenal glands. However, pancreatic metastasis resulting from thymic NEC is extremely uncommon, and only a few cases of patients with this disease have been reported. The current study presents the case of a patient with pancreatic metastasis resulting from thymic NEC. The patient was admitted to hospital with an anterior mediastinal neoplasm, which was identified using chest enhanced computed tomography. The patient underwent a monobloc excision of the tumor with resection of involved structures. Subsequently, a pathological diagnosis of atypical thymic carcinoid was provided, according to the morphological characteristics observed and the expression of neuroendocrine markers, as identified by immunohistochemistry. Following surgery, the patient received adjuvant chemotherapy and radiotherapy. However, $\sim 2$ years after surgery, metastasis at the pancreatic head was identified. The patient underwent a total pancreatectomy and splenectomy, and did not receive any post-operative therapies; however, the patient succumbed to the disease 9 months following surgery. Overall, the results from the present study demonstrate the clinical features of
\end{abstract}

Correspondence to: Professor Yongsheng Wang, Department of Medical Oncology, Cancer Center, State Key Laboratory of Biotherapy, West China Hospital, Sichuan University, 37 Guoxue Alley, Chengdu, Sichuan 610041, P.R. China

E-mail: cc300j@163.com

*Contributed equally

Key words: neuroendocrine carcinoma, pancreatic metastasis, thymic thymic NEC, which may aid with the diagnosis of this rare disease in other patients.

\section{Introduction}

Neuroendocrine tumors are neoplasms that originate from the cells of the endocrine and nervous systems. As rare tumors, they have an annual incidence of 2.5-5.0 cases per 100,000 individuals (1). The thymus is one of the rarest sites of neuroendocrine tumors. Thymic neuroendocrine carcinoma (NEC) was categorized as thymoma until 1972, when Rosai and Higa (2) differentiated thymic NEC from thymoma and other thymic carcinomas; thymic NEC is differentiated from thymoma microscopically, due to the presence of rosettes with central necrosis, and differentiation of neuroendocrine cells. Thymic NEC is an unusual neoplasm that accounts for only $2-4 \%$ of all anterior mediastinal neoplasms (3). The majority of thymic NECs have an aggressive biological behavior, including invasion, local recurrence and distant hematogenous metastasis (4). Distant metastasis is observed in various locations, including the bones, lungs, spleen, liver and adrenal glands $(5,6)$. However, pancreatic metastasis resulting from thymic NEC is extremely infrequent, and in the literature, to the best of our knowledge, there have only been a few cases of pancreatic metastasis from thymic NEC (7-9). The current study presents the case of a patient with rare pancreatic metastasis resulting from thymic NEC.

\section{Case report}

A 60-year-old man was admitted to the West China Hospital (Chengdu, China) in January 2012 with a 7.1x6.3-cm anterior mediastinal mass, which was observed on a routine chest enhanced computed tomography (CT; SOMATOM Definition Flash; Siemens Healthcare GmbH, Erlangen, Germany) scan (Fig. 1A). The medical history of the patient revealed tuberculous pleurisy, which occurred 40 years previously. A general physical examination was unremarkable. The patient underwent surgery to resect the mass. During the surgery, the tumor was observed to be located in the thymus with invasion to the adjacent structures, including the pericardium, aortic 

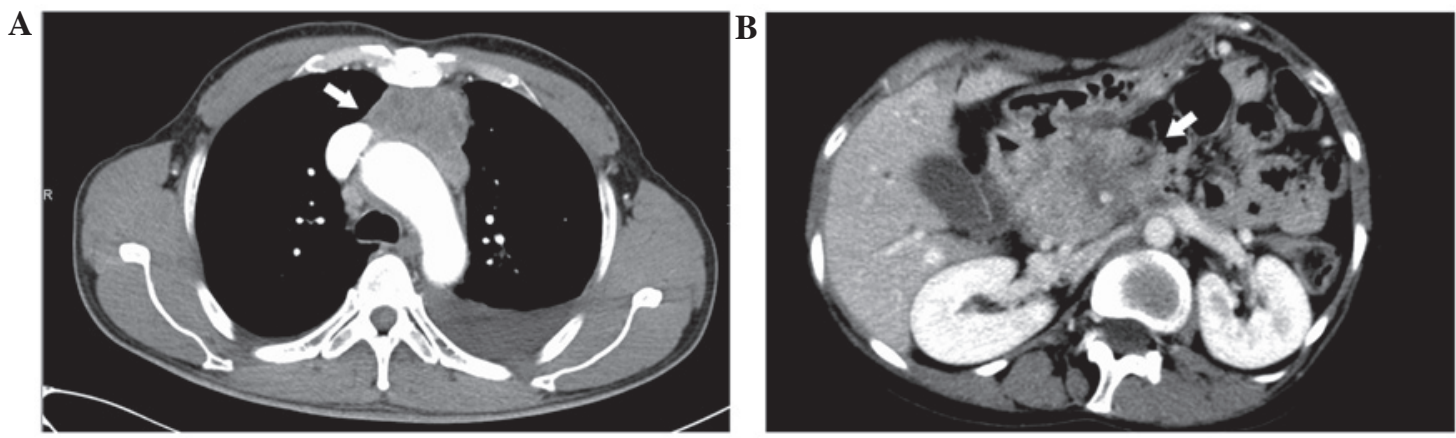

Figure 1. CT scans of the patient showing thymic neuroendocrine carcinoma with pancreatic metastasis. (A) Chest CT revealing the presence of a 7.1x6.3-cm mass located in the anterior mediastinum, as indicated by the white arrow. (B) Abdominal CT scan performed $\sim 2$ years later revealing the presence of a $4.5 \times 4.1-\mathrm{cm}$ mass located in the pancreatic head, as indicated by the white arrow. CT, computed tomography.
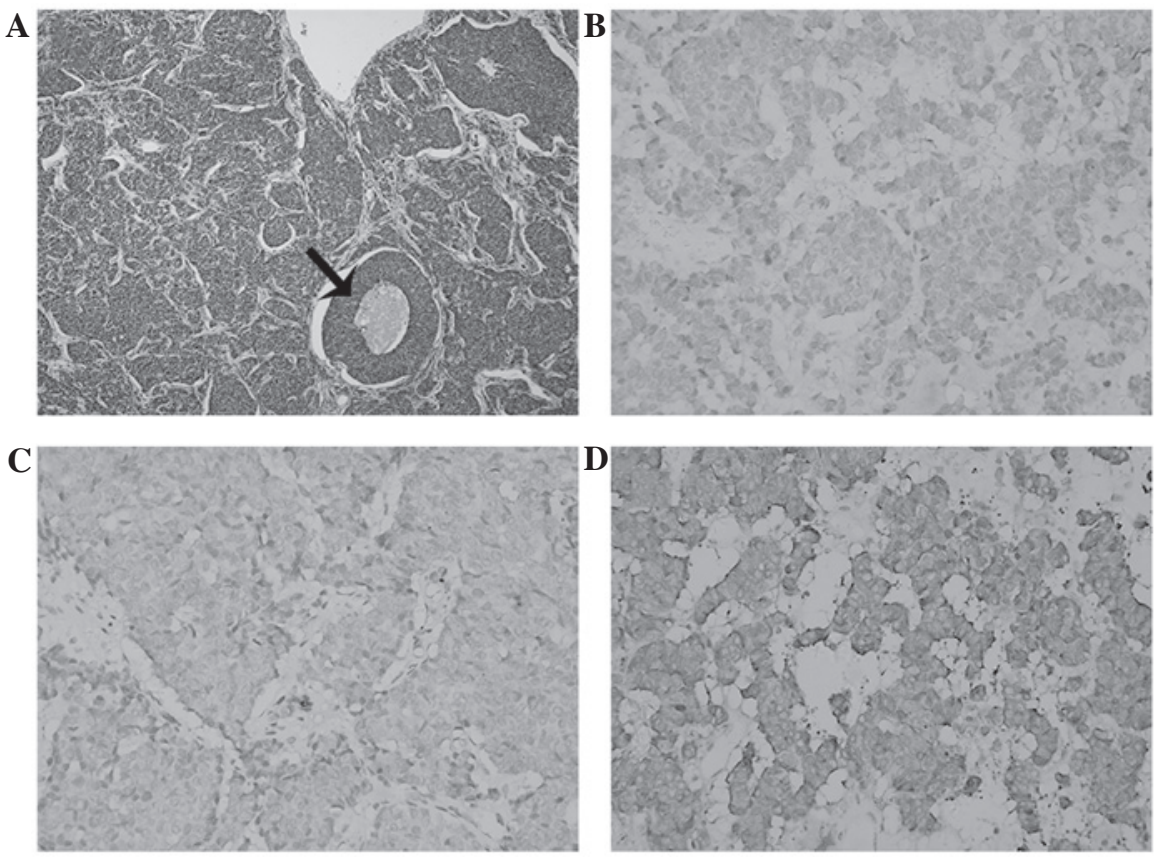

Figure 2. Original thymic tumor. (A) Thymic tumor cells were arranged in irregular, rosette-like acinar structures. Necrosis was observed, as indicated by the black arrow (hematoxylin and eosin staining; magnification, x100). Immunohistochemistry demonstrated that the thymic tumor cells expressed (B) neural cell adhesion molecule, (C) chromogranin A and (D) synaptophysin (magnification, $\mathrm{x} 400$ ).

arch and left brachiocephalic vein. Consequently, the patient underwent monobloc excision of the tumor with resection of the involved structures.

Macroscopically, the resected tumor was elastic and soft and measured $9.0 \times 7.0 \mathrm{~cm}$ in size. The cut surface of the tumor was gray with regions of hemorrhage and necrosis. A diagnosis of atypical thymic carcinoid was provided based on the morphological characteristics and necrosis observed using hematoxylin and eosin staining of formalin-fixed paraffin-embedded 5- $\mu \mathrm{m}$ thick tumor tissue sections (Fig. 2A). Immunohistochemistry was performed using 3,3'-diaminobenzidine staining (ZSGB-BIO, Beijing, China) and the following primary antibodies: Mouse monoclonal anti-human neural cell adhesion molecule (dilution, 1:100; catalog no., ZM-0057; ZSGB-BIO); rabbit monoclonal anti-human chromogranin A (dilution, 1:200; catalog no., ZA-0507; ZSGB-BIO); and rabbit polyclonal anti-human synaptophysin (dilution, 1:200; catalog no., RAB-0155; Maixin Biotech,
Fuzhou, China). The tissue sections were then visualized under a microscope (Eclipse E600; Nikon Corporation, Tokyo, Japan). Immunohistochemistry demonstrated that the tumor cells expressed neuroendocrine markers, including neural cell adhesion molecule (Fig. 2B), chromogranin A (Fig. 2C) and synaptophysin (Fig. 2D), which confirmed the diagnosis of an atypical thymic carcinoid. The Ki-67 index of the tumor cells was $\sim 20 \%$. The patient received post-operative treatment 4 weeks after surgery, which consisted of chemotherapy and radiotherapy. In total, the patient received 4 cycles of carboplatin ( $450 \mathrm{mg}$ on day 1 of a 4 -week cycle) and etoposide $(100 \mathrm{mg} /$ day on days 1-5). Subsequently, the mediastinal structures of the patients were irradiated with 27 fractions at a total dose of 54 Gy over 6 weeks. Follow-up was scheduled every 6 months, and consisted of clinical examination, CT scans and blood tests.

In November 2013, the patient was readmitted to hospital due to the presence of a tumor in the pancreatic head, 

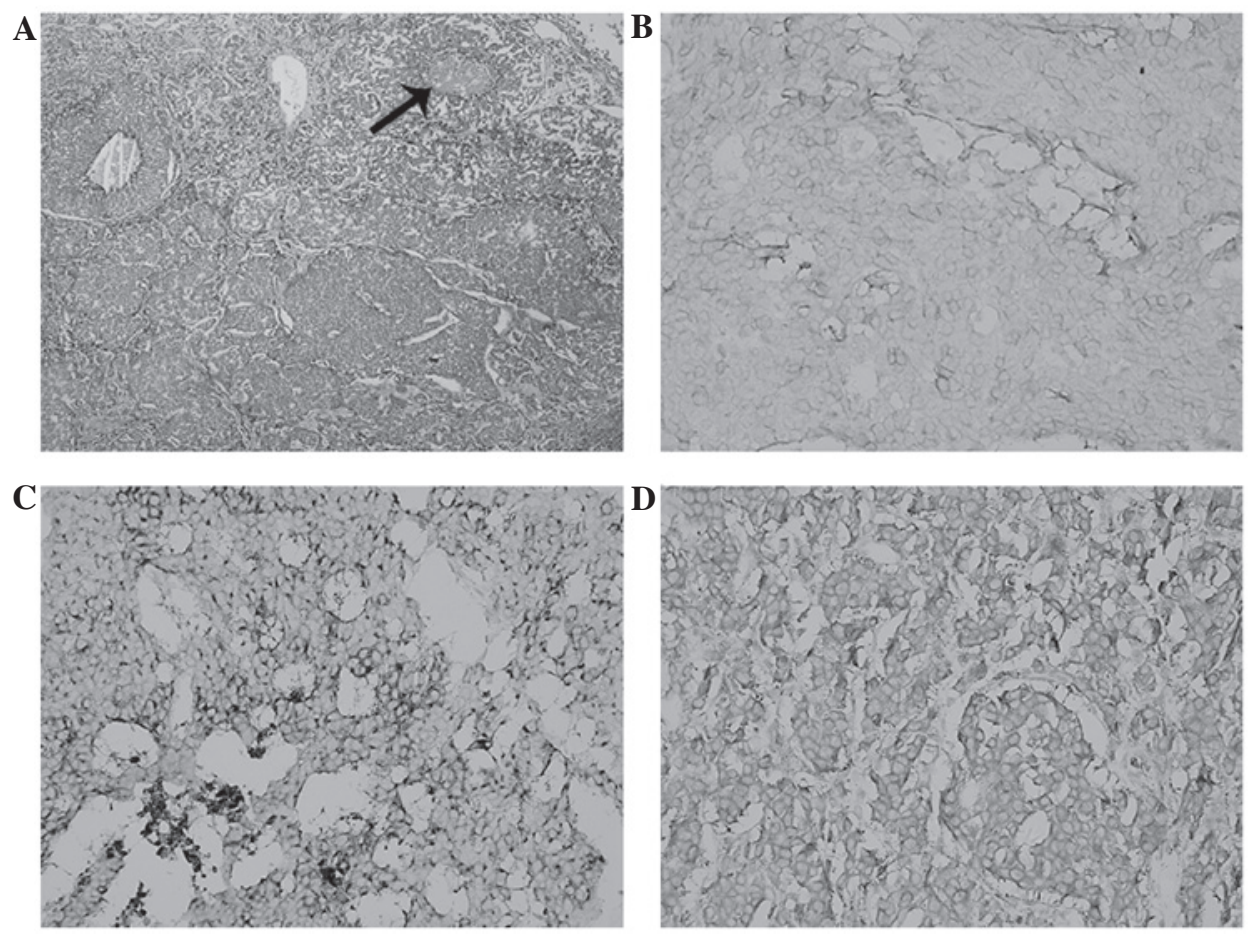

Figure 3. Pancreatic metastasis. (A) Metastatic tumour cells were similarly arranged in irregular, rosette-like acinar structures. Necrosis was observed, as indicated by the black arrow (hematoxylin and eosin staining; magnification, x100). Immunohistochemistry demonstrated that the metastatic tumor cells expressed (B) neural cell adhesion molecule, (C) chromogranin and (D) synaptophysin (magnification, $\mathrm{x} 400$ ).

which had been observed on a CT scan during follow-up (Fig. 1B). The patient underwent a total pancreatectomy and splenectomy to resect the pancreatic head tumor. Macroscopically, the resected tumor was elastic and soft, and measured $7.0 \times 6.0 \mathrm{~cm}$ in size. Histological examination revealed the presence of 1 peripancreatic tumoral nodule, and 2 out of 4 peripancreatic lymph nodes were positive for metastasis. Pathological examination revealed that the cytological morphology of the metastasis was identical to that of the originally resected thymic carcinoma (Fig. 3A). Immunohistochemistry revealed that the pancreatic tumor cells expressed neural cell adhesion molecule (Fig. 3B), chromogranin A (Fig. 3C) and synaptophysin (Fig. 3D); however, the tumor cells did not express insulin, gastrin, glucagon and somatostatin. The Ki-67 index of the cells was $10-25 \%$. A final diagnosis of pancreatic metastasis resulting from thymic NEC was formed. The patient did not receive post-operative therapies, and succumbed to the disease 9 months subsequent to surgery.

Written informed consent was obtained from the family of the patient for the publication of the present study.

\section{Discussion}

Thymic NEC is an extremely rare tumor that only accounts for $2-4 \%$ of all anterior mediastinal tumors and $\sim 6 \%$ of carcinoid tumors $(3,10)$. The majority of patients diagnosed with thymic NEC are in the 4th and 5th decades of life, and there is a $3: 1$ male to female ratio $(5,11,12)$. Symptomatic patients usually present with dyspnea, cough, chest pain and superior vena cava syndrome due to the presence of the mediastinal mass. In addition, clinical endocrinopathy develops in $\sim 50 \%$ of all patients; Cushing's syndrome (25\%) and multiple endocrine neoplasia type $1(15 \%)$ are the most common $(12,13)$. However, similar to the case presented in the current study, over one-third of patients are asymptomatic, and the tumors are incidentally observed on routine chest CT (4).

Thymic NEC is definitively diagnosed using immunohistochemistry. Thymic NEC cells express neuroendocrine markers, including synaptophysin, chromogranin A and neural cell adhesion molecule. In addition, thymic NEC is morphologically categorized into four main types: Typical carcinoid, atypical carcinoid, large cell neuroendocrine carcinoma and small cell carcinoma $(14,15)$. Therefore, according to the immunohistochemical and morphological diagnostic criteria, the patient in the present study was diagnosed with thymic atypical carcinoid.

Surgery is the most effective treatment for thymic NEC and radical excision is the most critical factor for predicting the long-term survival of patients (16). Radical excision often consists of en-bloc resection of the tumor with resection of the involved structures (17). The role of radiotherapy in the treatment of thymic NEC is controversial; however, it is recommended in order to prevent local recurrence of invasive tumors $(5,6,18)$. Single-agent or combination drug chemotherapies, including 5-fluorouracil, streptozotocin, carmustine, cisplatin and etoposide, have been used without any significant effect on the recurrence rate and overall survival time of patients $(11,19,20)$. However, although there is a limited effect on the long-term survival of patients, adjuvant chemotherapy may lead to mixed short-term survival times of patients. Therefore, a multidisciplinary therapy consisting of surgery, post-operative chemotherapy and radiotherapy is recommended to achieve the best outcome in patients with 
thymic NEC. In the current study, the patient underwent a complete resection and received post-operative chemotherapy and radiotherapy.

Despite multidisciplinary therapy, thymic NEC has a poor prognosis. Metastasis is common in thymic NEC; even though the patient in the current study had been treated with a radical excision, plus post-operative chemotherapy and radiotherapy, metastasis occurred in the pancreatic head. It is well known that the majority of thymic NECs possess a more aggressive biological behavior compared with other thymic epithelial tumors and neuroendocrine carcinoma originating at other locations, including the gastrointestinal tract and lungs (14). The aggressive biological behavior exhibited by thymic NEC may comprise invasion to proximal structures, including the pericardium, mediastinal fatty tissue, major blood vessels and lungs, local recurrence and distant hematogenous metastasis. Invasion to local structures is reported in $50 \%$ of cases of thymic NEC and distant hematogenous metastasis is reported in $20-30 \%$ of cases (21). Distant metastasis is identified in various locations, often in the bones, lungs, spleen, liver, brain and adrenal glands. However, in the current study, the patient exhibited an extremely rare pancreatic metastasis. To the best of our knowledge, there have only been 3 cases of patients with pancreatic metastasis resulting from thymic NEC previously reported in the literature (7-9). Axelson et al (7) reported a case in which several pancreatic tumors, which were located in the body and tail of the pancreas, were diagnosed 8 years following treatment for primary thymic NEC. In that study, the patient underwent a thymectomy and pancreatectomy, and 40 Gy of radiotherapy following the thymectomy. Lee et al (8) reported a case of pancreatic metastasis resulting from thymic NEC 2 years following therapy for a primary tumor. The patient received radiotherapy following a thymectomy and chemotherapy, which was administered to the patient subsequent to a pancreatectomy. Varytimiadis et al (9) also reported a case of pancreatic metastasis of thymic NEC, which was observed 5 years following treatment for a primary lesion. The patient in that study received radiotherapy subsequent to each surgery, and chemotherapy was administered to the patient following a thymectomy. Additional details concerning the therapeutic regimens, outcomes of patients and imaging results of the primary thymic NEC were not provided in these 3 studies.

Thymic NEC is extremely rare, and metastatic spread to the pancreas is particularly rare. Consequently, there is limited clinical experience available to formulate effective therapeutic regimens to treat thymic NEC. Therefore, more cases of patients with thymic NEC require documentation to aid in the study of this disease.

\section{References}

1. Oberg K and Castellano D: Current knowledge on diagnosis and staging of neuroendocrine tumors. Cancer Metastasis Rev 30 (Suppl 1): 3-7, 2011.
2. Rosai J and Higa E: Mediastinal endocrine neoplasm, of probable thymic origin, related to carcinoid tumor. Clinicopathologic study of 8 cases. Cancer 29: 1061-1074, 1972.

3. Wick MR and Rosai J: Neuroendocrine neoplasms of the mediastinum. Semin Diagn Pathol 8: 35-51, 1991.

4. Chaer R, Massad MG, Evans A, Snow NJ and Geha AS: Primary neuroendocrine tumors of the thymus. Ann Thorac Surg 74: 1733-1740, 2002

5. Fukai I, Masaoka A, Fujii Y, Yamakawa Y, Yokoyama T, Murase T and Eimoto T: Thymic neuroendocrine tumor (thymic carcinoid): A clinicopathologic study in 15 patients. Ann Thorac Surg 67: 208-211, 1999.

6. de Montpréville VT, Macchiarini P and Dulmet E: Thymic neuroendocrine carcinoma (carcinoid): A clinicopathologic study of fourteen cases. J Thorac Cardiovasc Surg 111: 134-141, 1996.

7. Axelson J, Kobari M, Furukawa T and Matsuno S: Thymic carcinoid in the pancreas: Metastatic disease or new primary tumours. Eur J Surg 165: 270-273, 1999.

8. Lee YT, Tse GM, Lai PB and Sung JJ: Metastatic thymic neuroendocrine carcinoma presenting as a pancreatic tumor. Endoscopy 38 (Suppl 2): E58-E59, 2006.

9. Varytimiadis K, Kalaitzakis E, Salla C, Ghika E, Pandazopoulou A and Karoumpalis I: Pancreatic metastasis of thymic neuroendocrine carcinoma: Is there a role for endoscopic ultrasound? Report of a case and review of the literature. Pancreas 38: 230-232, 2009.

10. Soga J, Yakuwa Y and Osaka M: Evaluation of 342 cases of mediastinal/thymic carcinoids collected from literature: A comparative study between typical carcinoids and atypical varieties. Ann Thorac Cardiovasc Surg 5: 285-292, 1999.

11. Filosso PL, Actis Dato GM, Ruffini E, Bretti S, Ozzello F and Mancuso M: Multidisciplinary treatment of advanced thymic neuroendocrine carcinoma (carcinoid): Report of a successful case and review of the literature. J Thorac Cardiovasc Surg 127: 1215-1219, 2004.

12. Detterbeck FC and Parsons AM: Thymic tumors. Ann Thorac Surg 77: 1860-1869, 2004.

13. Kondo K and Monden Y: Therapy for thymic epithelial tumors: A clinical study of 1,320 patients from Japan. Ann Thorac Surg 76: 878-884, discussion 884-885, 2003.

14. Moran CA and Suster S: Neuroendocrine carcinomas (carcinoid tumor) of the thymus. A clinicopathologic analysis of 80 cases. Am J Clin Pathol 114: 100-110, 2000.

15. Chetty R, Batitang S and Govender D: Large cell neuroendocrine carcinoma of the thymus. Histopathology 31: 274-276, 1997.

16. Kim DJ, Yang WI, Choi SS, Kim KD and Chung KY: Prognostic and clinical relevance of the World Health Organization schema for the classification of thymic epithelial tumors: A clinicopathologic study of 108 patients and literature review. Chest 127: 755-761, 2005.

17. Spaggiari L and Pastorino U: Double transmanubrial approach and sternotomy for resection of a giant thymic carcinoid tumor. Ann Thorac Surg 72: 629-631, 2001.

18. de Perrot M, Spiliopoulos A, Fischer S, Totsch M and Keshavjee S: Neuroendocrine carcinoma (carcinoid) of the thymus associated with Cushing's syndrome. Ann Thorac Surg 73: 675-681, 2002.

19. Wang DY, Chang DB, Kuo SH, Yang PC, Lee YC, Hsu HC and Luh KT: Carcinoid tumours of the thymus. Thorax 49: 357-360, 1994.

20. Gal AA, Kornstein MJ, Cohen C, Duarte IG, Miller JI and Mansour KA: Neuroendocrine tumors of the thymus: A clinicopathological and prognostic study. Ann Thorac Surg 72: 1179-1182, 2001.

21. Wick MR, Scott RE, Li CY and Carney JA: Carcinoid tumor of the thymus: A clinicopathologic report of seven cases with a review of the literature. Mayo Clin Proc 55: 246-254, 1980. 